\title{
Studies on Inhibiting Effect of Aminopterin and other Folic Acid Antagonist, and Folic Acid upon the Yoshida Sarcoma
}

\author{
By
}

\section{Takahiko Asano}

(胡野教展)

(From Pathological Laboratory of Prof. T. Yoshida, Faculty of Medicine, Tohoku University, Sendai)

(Recieived for publication, July 26, 1952)

Since attention had been called to the significant inhibiting effect of folic acid antagonists upon malignant growth, numerous researches of their inhibiting effect were made. ${ }^{1)}$ So far some researchers have reported on this inhibiting effect of the folic acid. However, the description of morphological changes of tumor cells through this substance does not seem sufficient. In the present studies I examined chiefly cytological effect of the folic acid and folic acid antagonists upon Yoshida sarcoma cells, taking into consideration their inhibiting effect upon the tumor growth.

Substances examined are as follows:-

1) Aminopterin

2) Methopterin

3) Folic acid

4) 2,4,5,6-tetraaminopyrimidine

5) 2-amino 4-hydrooxy 6,7-dimethylpteridine

6) 2,4-diamino 6,7-dimethylpteridine

7) 2,4-diamino 7 or 6-tetraoxybutylpteridine

8) 2,6-diaminopurine

9) 2,5,6-triamino 4-oxypyrimidine

10) 2-amino 4-oxy 6 or 7-phenylpteridine

11) 2,4-diamino 6 or 7-phenylpteridine

12) 2,4-diamino 6,7 (9,10-phenanthro) pteridine

13) 2,4-diamino 6,7-diphenylpteridine

14) 2,4-diamino 6 or 7 -( $p$-acetaminophenyl) pteridine

15) 2,4-diamino 6 or 7-( $p$-aminophenyl) pteridine

The compounds were supplied by the Iatrochemical Institute Tokyo, except aminopterin and methopterin which were offered from the Lederle Laboratories through Dr. Sugiura of the Sloan-Kettering Institute, New 
York City. Aminopterin supplied by the Iatrochemical Institute was also examined.

Experiments were carried out after the formula of our "Screeningtest " employing Yoshida Sarcoma animals.")

Results

1. Aminopterin: Table I indicates the survival days of tumor animals injected with aminopterin in different doses. Cases injected $1.0 \mathrm{mg}$., $0.5 \mathrm{mg}$., and $0.25 \mathrm{mg}$. died shortly after the injection from intoxication. In cases of $0.1 \mathrm{mg}$. injection, some died from intoxication, while others survived and died later from tumor invasion. Cases injected $0.05 \mathrm{mg}$. did not show any sign of intoxication and died from tumor invasion. Three of the four animals received a single injection of $0.05 \mathrm{mg}$. showed an evident prolongation of life (Table I).

The principal changes of tumor cells are degeneration of nuclei, such as caryorrhexis, caryolysis, etc., in resting tumor cells, but changes of chromosomes in mitotic tumor cells are not evident. Though the degeneration of cytoplasma is noted in cases of $0.05 \mathrm{mg}$. injection, it is most evident in cases of $1.0 \mathrm{mg}$. injection. It begins to appear from the 12. hour after injection and comes to the extreme after the 24. hour when in the majority of tumor cells as well as in normal monocytes and eosinoophile leucocytes in the ascites nuclear destructions are noted (Fig. 1-2). The neutrophile leucocytes do not appear from immediately after injection to the end. At the 48 . hour almost remaining tumor cells are remarkably increased in size (swelling) and their cytoplasma.indicates vacuolar degeneration (Figs. 3-6). At the 72. hour cytoplasma of tumor cells is stained so faintly that it is very hard to distinguish them from normal monocytes. At this time epistaxis and hemorrhagic diarrhoea begin to appear and the animals die in few days after that, i.e. about on the 7th day after the injection.

In case of $0.05 \mathrm{mg}$. injection, the degeneration of tumor cells appears slowly from the 24 . hour. At the 72 . hour, however, the peritoneal cavity has regained the pure culture state of actively proliferating tumor cells.

In cases of $0.03 \mathrm{mg}$, $0.02 \mathrm{mg}$., $0.01 \mathrm{mg}$., and $0.005 \mathrm{mg}$. injections the changes in tumor cells did not occur.

Since aminopterin is fairly toxic, it seems to expect any prolongation of life of tumor animals by the injection of this substance. However, in three of the four cases which received the twice repeated injection of 0.05 mg. aminopterin (Lederle) remarkable prolongation of life (Table I) was obtained. In many cases which received doses $0.1 \mathrm{mg}$. anemia, epistaxis, and hemorrhagic diarrhoea appeared about 4 days after injection. 
TABLE I

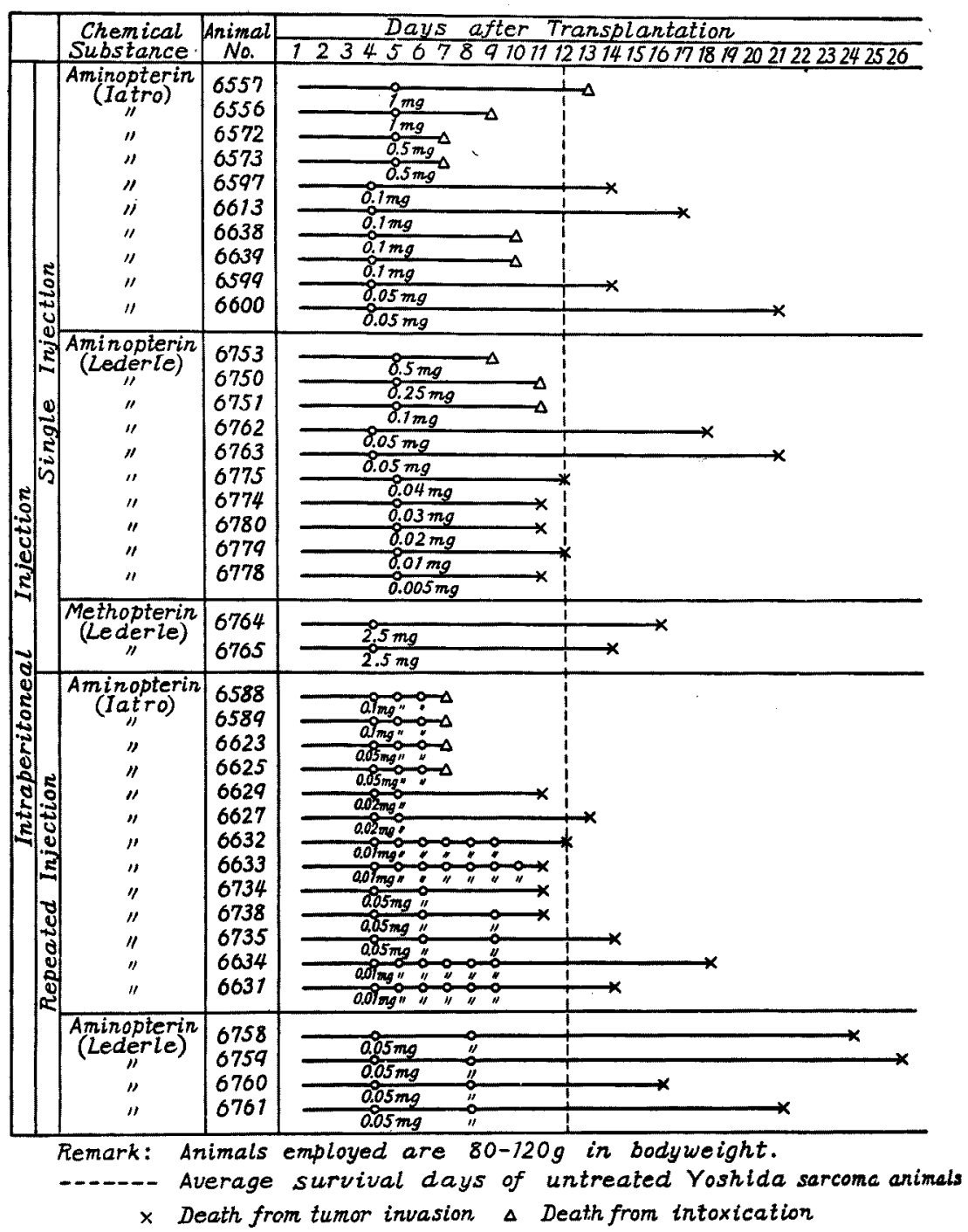

Subcutaneous injection of the substance presented the almost same cytological effect as intraperitoneal injection upon the tumor cells in the ascites.

2. Methopterin: Injection of the substance $2.5 \mathrm{mg}$. (Lethal dose is $5 \mathrm{mg}$. per $100 \mathrm{~g}$. body weight) produced the almost same cellular changes as above described.

3. Twelve other folic acid antagonists and folic acid demonstrated 
Fig. 1

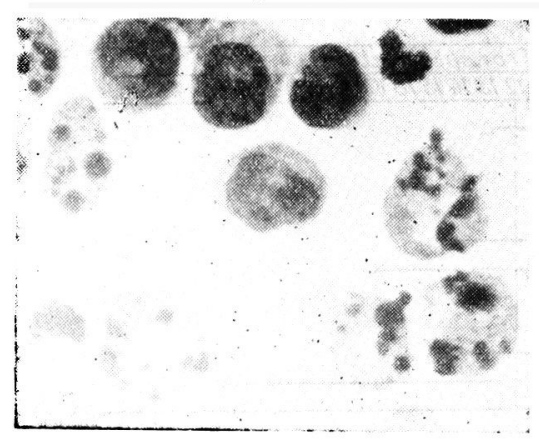

Fig. 3

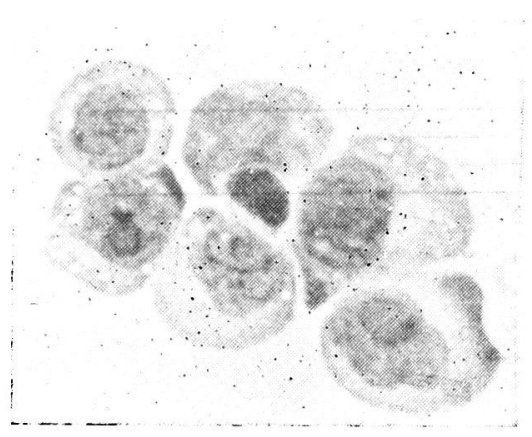

Fig. 5

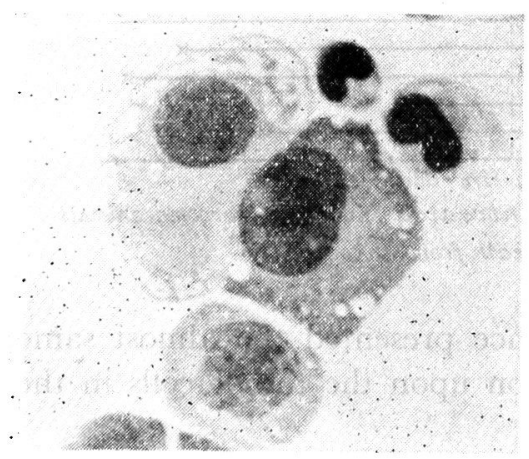

Fig. 2

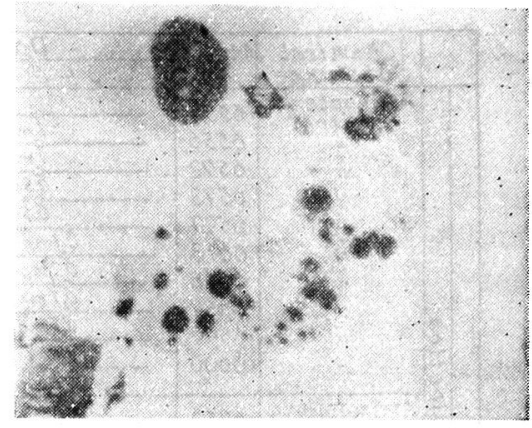

Fig. 4

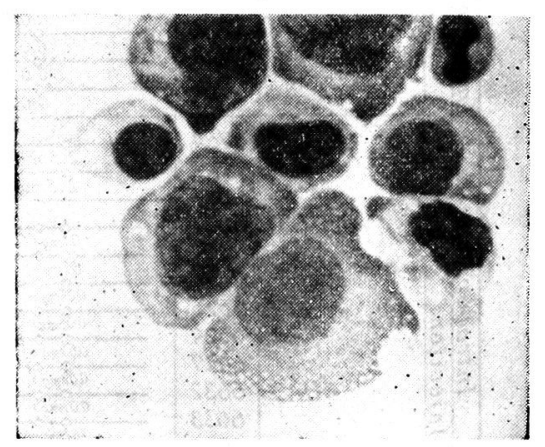

Gig. 6

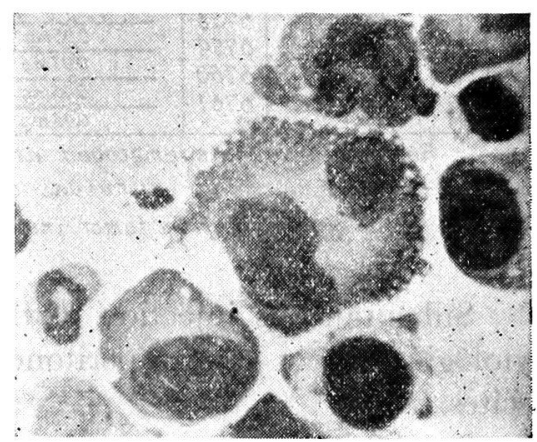

Figs. 1-2. 2 days after injection of $1.0 \mathrm{mg}$. aminopterin: Caryorrhexis of tumor cells.

Figs. 3-6. 2 days after injection of $1.0 \mathrm{mg}$. aminopterin: Swelling and vacuolar degeneration of cytoplasma, and faintly stained nuclei. 
neither notable cellular changes nor inhibiting effect even in cases given the half lethal dose or the lethal dose.

\section{SUMmary}

1. Aminopterin demonstrated a notable inhibiting effect upon the growth of the Yoshida sarcoma, especially in cases which received the repeated intraperitoneal injection of $0.05 \mathrm{mg}$. per $100 \mathrm{~g}$. body weight. Non-specific changes in the tumor cells after the injection, such as caryorrhexis and vacuolar degeneration were described. These cellular changes were obtained also by subcutaneous injection of the compound.

2. Other 13 folic acid antagonists including Methopterin and folic acid demonstrated neither remarkable cellular changes nor inhibiting effect.

Acknowledgements. The author wishes to express his gratefulness to the Lederle Laboratories, Dr. Sugiura, and the Iatrochemical Institute for supplying the compounds which made this study possible. This study was supported by the "Grant in Aid for Fundamental Scientific Research" of the Ministry of Education.

\section{References}

1) Thiersch J.B. \& Philips, F.S.: Am. J. Med. Sci. 1949, 217, 575-585.

2) Yoshida, Tomizo: Gann 1950, 41, 93-96. 